\title{
NDNF inhibits the migration and invasion of human renal cancer cells through epithelial-mesenchymal transition
}

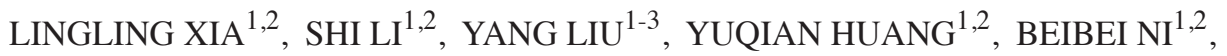

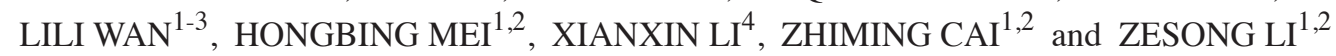 \\ ${ }^{1}$ Guangdong Key Laboratory of Systems Biology and Synthetic Biology for Urogenital Tumors; \\ ${ }^{2}$ Shenzhen Key Laboratory of Genitourinary Tumor, Shenzhen Second People's Hospital, First Affiliated Hospital of \\ Shenzhen University, Shenzhen, Guangdong 518000; ${ }^{3}$ Department of Oncology, Guangzhou Medical University, Guangzhou, \\ Guangdong 510182; ${ }^{4}$ Department of Urology, Peking University Shenzhen Hospital, Shenzhen, Guangdong 518036, P.R. China
}

Received April 4, 2018; Accepted November 21, 2018

DOI: $10.3892 /$ ol.2019.9937

\begin{abstract}
Neuron-derived neurotrophic factor (NDNF) is a glycosylated, disulfide-bonded secretory protein that contains a fibronectin type III domain. NDNF has been identified as a neurotrophic factor; however, its role in carcinogenesis has not yet been identified. To investigate the expression and role of NDNF in carcinogenesis, the expression of NDNF in human Renal cell carcinoma (RCC) cell lines and tissues was detected by reverse transcription-quantitative polymerase chain reaction (RT-qPCR) and western blot analysis. Cell proliferation was investigated using CCK-8 and colony formation assays, and the cell invasion and immigration capacity was evaluated using the transwell assay. The results demonstrated that NDNF expression was downregulated in RCC cell lines and RCC tissues. Restoring NDNF expression significantly inhibited the proliferation, migration and invasion of RCC cells. The study also demonstrated that the inhibitory effect of NDNF on invasive ability was mediated by suppressing the epithelial-mesenchymal transition (EMT) in RCC cells. NDNF may therefore be considered an important regulator of EMT in RCC progression and may represent a novel promising target for antimetastatic therapy.
\end{abstract}

\section{Introduction}

Renal cell carcinoma (RCC) accounts for $80 \%$ of all primary renal neoplasms and comprises $2-3 \%$ of all malignancies (1).

Correspondence to: Professor Zesong Li, Guangdong Key Laboratory of Systems Biology and Synthetic Biology for Urogenital Tumors, Shenzhen Second People's Hospital, First Affiliated Hospital of Shenzhen University, 3002 Sungang West Road, Futian, Shenzhen, Guangdong 518000, P.R. China

E-mail:1zssc@yahoo.com

Abbreviations: NDNF, neuron-derived neurotrophic factor; RCC, Renal cell carcinoma; EMT, epithelial-mesenchymal transition

Key words: RCC, NDNF, EMT, migration, invasion
Partial nephrectomy is the most effective treatment for local clear cell RCC (ccRCC); however, 30\% of patients develop metastatic disease after surgery. Although some kinase inhibitors have been used in clinical applications, metastatic RCC remains largely incurable due to the off-target effects of current agents, and resistance to chemotherapy and radiation $(2,3)$. Therefore, the identification of novel markers and targets for the early detection and treatment of RCC is required (4-7).

Neuron-derived neurotrophic factor (NDNF) is a glycosylated, disulfide-bonded secretory protein that contains a fibronectin type III domain. NDNF has been identified as a neurotrophic factor that can promote neuron migration, growth and survival, as well as neurite outgrowth (8). Furthermore, NDNF promotes endothelial cell survival and vessel formation, and has an important role in the process of revascularization through nitric oxide synthase 3-dependent mechanisms (9). NDNF can also exert favorable effects on various ischemic cardiovascular disorders (10); however, the functional role of NDNF in carcinogenesis has not yet been identified.

In the present study, the expression and roles of NDNF in RCC were investigated. The results demonstrated that NDNF was decreased in ccRCC. In addition, restoring NDNF expression inhibited the proliferation, migration and invasion of RCC via the suppression of epithelial-mesenchymal transition (EMT), which contributes to the progression of cancer by promoting loss of cell-cell adhesion, leading to a shift in cytoskeletal dynamics (11). NDNF may therefore be considered an important regulator of EMT in RCC progression.

\section{Materials and methods}

Patient samples. The present study included 69 samples from patients diagnosed with ccRCC. Renal cancer tissues and paired normal renal tissues were obtained from each patient and stored at $-80^{\circ} \mathrm{C}$. Written informed consent was provided by the patients and their families for the use of tissue specimens. The present study was approved by the Ethics Committee of Shenzhen Second People's Hospital (Shenzhen, China).

Cell culture and lentiviral infection. The human RCC cell lines ACHN, Caki-1, OS-RC-2, 786-O and 769-P, and the papillary 
RCC cell line Caki-2, were purchased from the American Type Culture Collection (Manassas, VA, USA), ACHN was cultured in MEM (Gibco; Thermo Fisher Scientific, Inc., Waltham, MA, USA), Caki-1 and Caki-2 were cultured in McCoy's 5A (Gibco; Thermo Fisher Scientific, Inc.), OS-RC-2, 786-O and 769-P were cultured in RPMI 1640 (Gibco; Thermo Fisher Scientific, Inc.). Cell lines were supplemented with 10\% FBS (Gibco; Thermo Fisher Scientific. Inc.), $100 \mathrm{U} / \mathrm{ml}$ penicillin (Gibco; Thermo Fisher Scientific, Inc.) and $100 \mu \mathrm{g} / \mathrm{ml}$ streptomycin (Gibco; Thermo Fisher Scientific, Inc.) and were grown in $37^{\circ} \mathrm{C}$, $5 \% \mathrm{CO}_{2}$ atmosphere. The lentiviral plasmids pLVX-3FLAG (Lenti-Control) and pLVX-NDNF-3FLAG (Lenti-NDNF) were designed by Shanghai GeneChem Co., Ltd. (Shanghai, China). The lentiviruses were generated by cotransfecting the $20 \mu \mathrm{g}$ lentiviral plasmid, $15 \mu \mathrm{g}$ pHelper1.0 (gag/pol element) and $10 \mu \mathrm{g}$ Helper2.0 (VSVG element) into 70-80\% confluence 293T cells (ATCC, Manassas, VA, USA) using Lipofectamine ${ }^{\circledR}$ 2000 (Invitrogen; Thermo Fisher Scientific, Inc., Waltham, MA, USA). After $48 \mathrm{~h}$ transfection, the viral supernatants were collected, centrifuged at $70,000 \times \mathrm{g}$ at $4^{\circ} \mathrm{C}$ for $2 \mathrm{~h}$ and filtered. $\mathrm{ACHN}$ and 786-O cells were infected with lentiviruses and $8 \mu \mathrm{g} / \mathrm{ml}$ polybrene (Sigma-Aldrich, Merck KGaA, Darmstadt, Germany). Infected cells were then exposed to $4 \mu \mathrm{g} / \mathrm{ml}$ puromycin (cat no. s7417; Selleck Chemicals, Houston, TX, USA) for 4 days. Single-cell clones with stable NDNF expression were identified by reverse transcription-quantitative polymerase chain reaction (RT-qPCR) and western blotting.

$R T$-qPCR. RT-qPCR was carried out as described previously $(12,13)$. Briefly, total RNA was extracted using TRIzol ${ }^{\circledR}$ reagent (Invitrogen; Thermo Fisher Scientific, Inc.) and cDNA was converted from total RNA using the PrimeScript ${ }^{\mathrm{TM}}$ RT reagent with gDNA Eraser (Takara Bio, Inc., Otsu, Japan) according to the manufacturer's protocol. RT-qPCR was performed by using HotStarTaq DNApolymerase and buffer (Quiagen GmbH, Hilden, Germany) with an initial denaturation period at $95^{\circ} \mathrm{C}$ lasting for $15 \mathrm{~min}$, followed by 35 cycles at $94^{\circ} \mathrm{C}$ for $45 \mathrm{sec}$, annealing at $60^{\circ} \mathrm{C}$ for $30 \mathrm{sec}$, and extension at $72^{\circ} \mathrm{C}$ for $45 \mathrm{sec}$ in a thermal cycler (Applied Biosystems; Thermo Fisher Scientific, Inc.). RT-qPCR was performed using SYBR Premix Ex Taq ${ }^{\mathrm{TM}}$ II (Takara Bio, Inc.) with the following cycling conditions: Initial denaturation at $95^{\circ} \mathrm{C}$ or $10 \mathrm{~min}$, followed by 40 cycles of denaturation at $95^{\circ} \mathrm{C}$ for $45 \mathrm{sec}$ and annealing at $60^{\circ} \mathrm{C}$ for $30 \mathrm{sec}$. The primer sequences were as follows: NDNF, forward 5'-GGGAAGGCTCAGGTG ATCTGGAAC-3', reverse 5'-AAACCGGATGGGGAACTA GACGAT-3'; and $\beta$-actin, forward 5'-GGCACCACACCT TCTACAATGAG-3', and reverse 5'-GGATAGCACAGCCTG GATAGCA-3'. Each sample was analyzed in triplicate using the ABI 7300 Real-Time PCR system (Applied Biosystems; Thermo Fisher Scientific, Inc.). The relative expression levels of NDNF were calculated using the $2^{-\Delta \Delta \mathrm{Cq}}$ method, as previously described (14).

Western blotting. Proteins were extracted from the cells using $1 \mathrm{ml}$ radioimmunoprecipitation assay buffer (Thermo Fisher Scientific, Inc.) containing $10 \mu$ l protease inhibitor cocktail (Sigma-Aldrich, Merck KGaA). Protein concentration was quantified using the bicinchoninic acid assay kit (Pierce; Thermo Fisher Scientific, Inc.) Proteins (40 $\mu \mathrm{g} / \mathrm{sample})$ were separated by $10 \%$ SDS-PAGE and transferred onto polyvinylidene difluoride membranes (EMD Millipore, Billerica, MA, USA). Membranes were blocked in 5\% skimmed milk at room temperature for $1 \mathrm{~h}$ and were incubated with the following primary antibodies diluted in PBS with 5\% bovine serum albumin (cat no. 4240GR100; NeoFROXX GmbH, Hesse, German) overnight at $4^{\circ} \mathrm{C}$ : NDNF (1:1,000 dilution, cat no. sc-242196; Santa Cruz Biotechnology, Inc., Dallas, TX, USA), $\beta$-tubulin (1:5,000 dilution, cat no. ab6046; Abcam, Cambridge, UK), N-cadherin (1:1,000 dilution, cat no. sc-7939; Santa Cruz Biotechnology, Inc.), E-cadherin (1:1,000 dilution, cat no. sc-31020; Santa Cruz Biotechnology, Inc.), Vimentin (1:1,000 dilution, cat no. sc-7557-R; Santa Cruz Biotechnology, Inc.), MMP-7 (1:1,000 dilution, cat no. sc-8832, Santa Cruz Biotechnology), Cyclin D1 (1:1,000 dilution, cat no. 2978, Cell Signaling Technology, Inc., Danvers, MA, USA) and $\beta$-catenin (1:1,000 dilution, cat no. sc-7963; Santa Cruz Biotechnology, Inc.). After three washes with TBST [20 mM Tris- $\mathrm{HCl}$ (pH 7.5), $150 \mathrm{mM} \mathrm{NaCl}, 0.1 \%$ Tween-20], membranes were incubated goat anti-rabbit (1:1,000 dilution, cat no. sc-2004, Santa Cruz Biotechnology, Inc.), rabbit-anti-goat (1:1,000 dilution, cat no. sc-2922, Santa Cruz Biotechnology, Inc.), or horse anti-mouse $(1: 1,000$ dilution, cat no. 7076P2, Cell Signaling Technology, Inc.). At room temperature for $1 \mathrm{~h}$. Bands were detected using the Alliance Imaging system (Uvitec, Cambridge, UK) with the Immobilon Western Chemiluminescent HRP Substrate (EMD Millipore).

Immunofluorescence. Immunofluorescence was carried out as previously described (11). Briefly, $2 \times 10^{3}$ cells were fixed in ice-cold $4 \%$ paraformaldehyde containing $0.1 \%$ Triton X-100 for $5 \mathrm{~min}$, and incubated with a mouse anti-flag monoclonal antibody (1:5,000 dilution; cat no. ABIN3020559, Abgent Biotech Co., Ltd., Suzhou, China) at $4^{\circ} \mathrm{C}$ overnight. Cells were then washed three times with PBS and incubated with a donkey anti-rabbit immunoglobulin $\mathrm{G}$ secondary antibody conjugated to Alexa Fluor ${ }^{\circledR} 647$ (1:1,000 dilution, cat no. A32795 Thermo Fisher Scientific, Inc.). Cell nuclei were stained with 4',6-diamidino-2-phenylindol (Sigma-Aldrich; Merck KGaA, Darmstadt, Germany). Immunofluorescence was visualized using a confocal fluorescence microscope.

NDNF ELISA. To detect extracellular NDNF released by ACHN and 786-O cells, $2 \times 10^{6}$ cells were cultured in MEM (Gibco; Thermo Fisher Scientific, Inc.) and O RPMI 1640 (Gibco) supplemented with 1\% FBS (Gibco; Thermo Fisher Scientific, Inc.) for $24 \mathrm{~h}$. The supernatant was collected and concentrated by ultrafiltration at $1,000 \mathrm{x} \mathrm{g}$ at room temperature for $20 \mathrm{~min}$. Subsequently, $10 \mu \mathrm{l}$ aliquots were analyzed using the commercially available NDNF ELISA kit (cat no. SEU433 Hu; Cloud-Clone Corp., Katy TX, USA), according to the manufacturer's protocol. Each ELISA plate was read on a microplate reader at $450 \mathrm{~nm}$ absorbance, with a correction wavelength of $540 \mathrm{~nm}$.

Cell proliferation and colony formation assays. Cell proliferation was assessed using the Cell Counting Kit-8 assay (Beyotime Institute of Biotechnology). Lentivirus-infected cells were grown in a 96 -well plate at $2 \times 10^{3}$ cells/well. After $0,24,48,72$ and $96 \mathrm{~h}$, the CCK- 8 reagent $(10 \mu \mathrm{l})$ was added to 

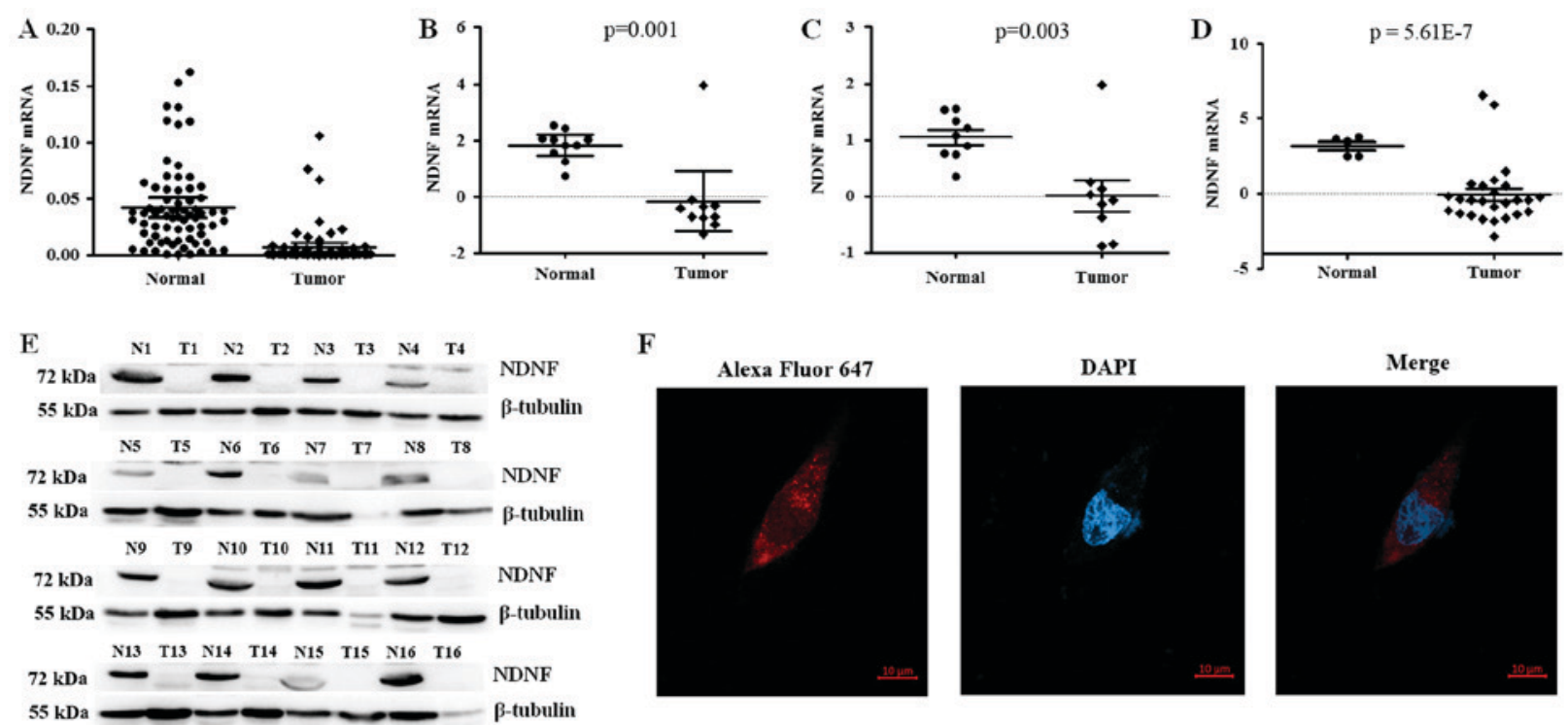

Figure 1. NDNF expression is reduced in ccRCC. (A) NDNF expression in 69 ccRCC and paired noncancerous tissues was determined by reverse transcription-quantitative polymerase reaction $(\mathrm{P}<0.0001)$. The Oncomine database was used to analyze NDNF expression in previously published RCC datasets: (B) Gumz et al (16), (C) Lenburg et al (17) and (D) Yusenko et al (18) using standard settings. (E) NDNF protein in ccRCC and paired healthy tissues, as determined by western blotting. (F) Immunofluorescence staining captured by fluorescence microscopy, including Alexa Fluor ${ }^{\circledR}$ 647, DAPI and merged images. The NDNF protein was observed mainly in the cytoplasm. Scale bar, $10 \mu \mathrm{m}$. ccRCC, clear cell RCC; DAPI, 4',6-diamidino-2-phenylindol; NDNF, neuron-derived neurotrophic factor; RCC, Renal cell carcinoma.

each well and incubated at $37^{\circ} \mathrm{C}$ for $2 \mathrm{~h}$. Then, the absorbance at $450 \mathrm{~nm}$ was detected using a microplate reader (Bio-Rad Laboratories Inc., Hercules, CA, USA). With regards to the colony formation assays, $1 \times 10^{3}$ infected ACHN and 786-O cells were seeded in a 6-well plate and cultured in MEM (Gibco; Thermo Fisher Scientific, Inc.) and RPMI 1640 (Gibco; Thermo Fisher Scientific, Inc.) for 2 weeks, respectively. Cells were then stained with $0.1 \%$ crystal violet for 5 min after fixation with $4 \%$ paraformaldehyde for $20 \mathrm{~min}$ at room temperature. Images of the results were captured, and the number of colonies was counted. Each experiment was performed in triplicate.

Cell migration and invasion assays. Cell migration was measured using 24-well Transwell chambers (pore size, $8 \mu \mathrm{m}$; Corning Incorporated, Corning, NY, USA). In total, $1 \times 10^{5}$ cells/well were seeded onto the upper chamber in serum-free medium. With regards to the cell invasion assay, $\sim 2 \times 10^{5}$ cells/well were seeded onto the upper chamber, which was coated with Matrigel (1:8; $50 \mu \mathrm{l} /$ well; BD Biosciences, San Jose, CA, USA). The lower chamber was filled with MEM (Gibco; Thermo Fisher Scientific, Inc.) medium for ACHN and RPMI 1640 (Gibco; Thermo Fisher Scientific, Inc.) medium for $786-O$ containing $10 \%$ FBS. After $48 \mathrm{~h}$, the cells on the surface of the upper chamber were wiped with cotton buds, whereas the cells on the bottom chamber were fixed in $4 \%$ paraformaldehyde for $15 \mathrm{~min}$ and stained with $0.1 \%$ crystal violet for $20 \mathrm{~min}$ at room temperature. Images of cell migration and invasion were captured using an inverted microscope. Eventually, cells contained in each chamber were removed using $1 \mathrm{ml} \mathrm{33 \%}$ acetic acid and seeded into 96-well plates, after which, the absorbance was measured at $570 \mathrm{~nm}$ with a microplate reader. Each experiment was performed at least three times.
Database mining. The Oncomine database (15) (https://www.oncomine.org/) was used for retrieving the alterations in NDNF mRNA levels in RCC tissues and corresponding disease-free normal and/or normal adjacent tissues. Data from Gumz et al (16), (C) Lenburg et al (17) and (D) Yusenko et al (18) were retrieved using standard settings.

Statistical analysis. All the data are presented as the means \pm standard deviation obtained from three separate experiments. Statistical analysis was conducted using GraphPad Prism 5.0 (GraphPad Software, San Diego, CA, USA) and SPSS 22.0 software (SPSS, Chicago, IL, USA). Paired t-tests were used to analyze the expression levels of NDNF in ccRCC compared with in adjacent normal kidney tissues, and nonpaired t-tests were used to determine the associations between NDNF expression in ccRCC compared to that in normal kidney tissues. Nonpaired t-tests were used to determine the effects of ectopic expression on cell function. $\mathrm{P}<0.05$ was considered to indicate a statistically significant difference.

\section{Results}

NDNF expression is reduced in ccRCC. In our previous study, deep sequencing analysis demonstrated that NDNF expression is decreased in ccRCC compared with in paired nontumor tissues (19). To investigate whether NDNF expression was altered during RCC carcinogenesis, NDNF expression in 69 ccRCC and paired nontumor tissues was analyzed. Results demonstrated that NDNF expression was silenced or strongly decreased in 65 of 69 ccRCC samples ( $\mathrm{P}<0.001$; Fig. 1A). An analysis of the Oncomine database also revealed that NDNF expression was significantly lower in RCC tissues compared with in normal renal tissues in three independent studies 
A

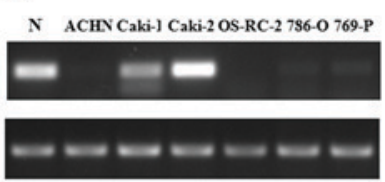

D

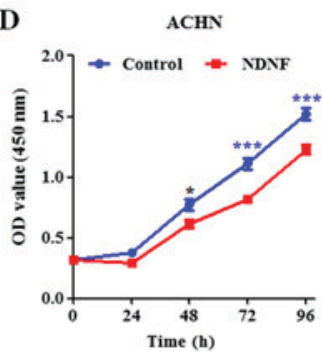

B

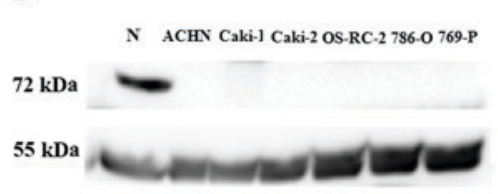

E

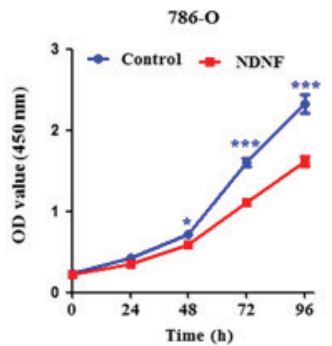

C

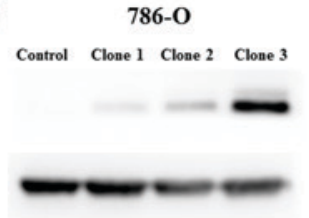

F

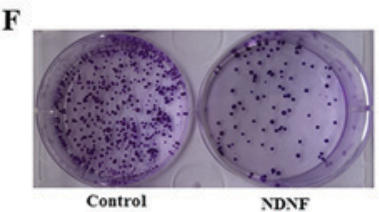

G

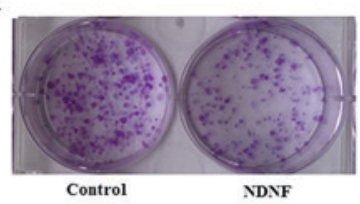

ACHN
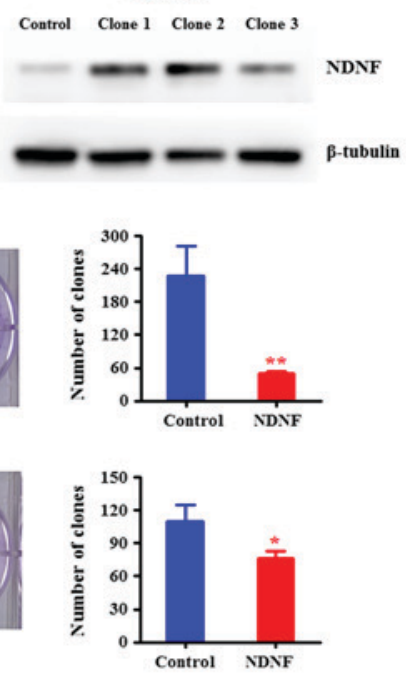

Figure 2. NDNF suppresses the proliferation of RCC cells. (A) Expression levels of NDNF mRNA in normal kidney tissues and various RCC lines (ACHN, Caki-1, Caki-2, 769-P, OS-RC-2 and 786-0) were determined by RT-PCR. (B) NDNF protein expression in RCC cell lines was determined by western blotting. (C) NDNF protein expression in single-cell clones infected with NDNF, and in control cells, was determined by western blotting. $\beta$-tubulin was used as an internal control. (D and E) Effects of ectopic NDNF expression on ACHN (clone 2) and 786-O (clone 3) cells; proliferation was detected using the Cell Counting Kit-8 assay. Experiments were repeated three times. Results are presented as the means $\pm \mathrm{SD}\left({ }^{*} \mathrm{P}<0.05,{ }^{* * * *} \mathrm{P}<0.001\right)$. (F and G) Effects of ectopic NDNF expression on the colony formation of ACHN (clone 2) and 786-O (clone 3) cells. NDNF-stable cells and control cells were seeded at low density and cultured for 2 weeks. Experiments were repeated three times. Results are presented as the means $\pm \mathrm{SD}\left(\mathrm{n}=3,{ }^{*} \mathrm{P}<0.05,{ }^{*} \mathrm{P}<0.01\right)$. NDNF group: Lenti-NDNF infected cells; Control group: Lenti-Control infected cells. NDNF, neuron-derived neurotrophic factor; OD, optical density; RCC, Renal cell carcinoma; SD, standard deviation.

(Fig. 1B-D) (16-18), thus indicating that NDNF was reduced in ccRCC. NDNF protein was also examined by western blotting in $16 \mathrm{RCC}$ clinical specimens and paired nontumor tissues. NDNF expression in RCC was markedly reduced compared with in paired nontumor tissues (Fig. 1E). These data revealed that the loss of NDNF expression may be associated with RCC carcinogenesis. Since the NDNF antibody used for western blotting was not suitable for immunofluorescence, NDNF expression and subcellular location in renal tissues were not determined. However, after the infection of ACHN cells with NDNF-3Flag expression lentiviruses, immunofluorescence analysis revealed the cytoplasmic location of NDNF-3Flag (Fig. 1F).

Analysis of NDNF expression in human RCC cell lines. The expression of NDNF in five RCC cell lines (ACHN, OS-RC-2, Caki-1, 786-O and 769-P) and in the Caki-2 papillary RCC cell line was determined. The results revealed that Caki-1 and Caki-2 cell lines expressed high NDNF mRNA expression levels, whereas no NDNF mRNA was detected in ACHN, OS-RC-2, 786-O and 769-P cell lines (Fig. 2A). NDNF protein expression levels were also examined in the RCC cell lines by western blotting. Notably, no NDNF protein was detected in the six cell lines while NDNF protein is high in normal kidney tissues, although Caki-1 and Caki-2 cells exhibited high NDNF mRNA levels (Fig. 2B). It has been reported that NDNF is a glycosylated, disulfide-bonded secretory protein and was detected in cell supernatants (8). However, no NDNF was detected in any of the RCC cell line supernatants (data not shown).

NDNF suppresses proliferation and colony formation of RCC cells. Since ACHN and 786-O RCC cell lines exhibited the lowest mRNA and protein expression levels (Fig. 2A and B), NDNF expression was reintroduced into human ACHN and 786-O cell lines. RCC infection with NDNF-bearing lentiviral or empty vectors (control) was performed. The infected cells were subsequently selected with puromycin, and single-cell clones were selected to establish stable cell lines. The ectopic expression of exogenous NDNF was confirmed by RT-qPCR analysis (data not shown) and western blotting (Fig. 2C). Only a very small amount of NDNF was detected in the cell culture supernatant (data not shown). Clones 2 and 3 for 786-O, and clones 1 and 2 for ACHN presented high NDNF expression, and were therefore selected for further analysis.

The effects of NDNF on RCC were then assessed by measuring cell proliferation and colony formation. The results revealed that ectopic expression of NDNF significantly reduced the proliferation of ACHN (clone 2) and 786-O (clone 3 ) in a time-dependent manner $(\mathrm{P}<0.05)$ (Fig. 2D and $\mathrm{E})$. The colony formation assay also revealed that ectopic expression of NDNF significantly decreased ACHN (clone 2) (Fig. 2F) and 786-O (clone 3) (Fig. 2G) colony formation. Similar results were obtained from ACHN (clone 1) and 786-O (clone 2) cells (data not shown).

NDNF inhibits the migration and invasion of RCC cells. To further investigate whether NDNF affects RCC cell migration and invasion, Transwell migration and invasion assays were performed. Compared with control cells, ectopic expression of NDNF significantly suppressed the migratory abilities of ACHN (clone 2) and 786-O (clone 3) cells (Fig. 3A-D). The Boyden chamber tests demonstrated that the invasive abilities of ACHN (clone 2) and 786-O (clone 3) cells transfected with NDNF were decreased compared 
A

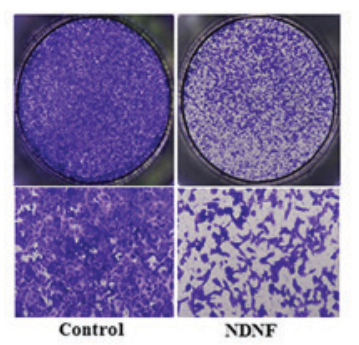

$\mathbf{E}$

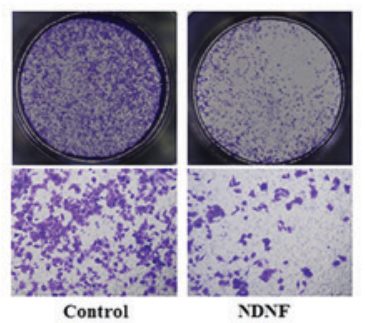

ACHN B

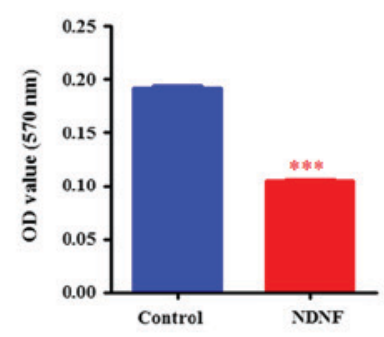

F

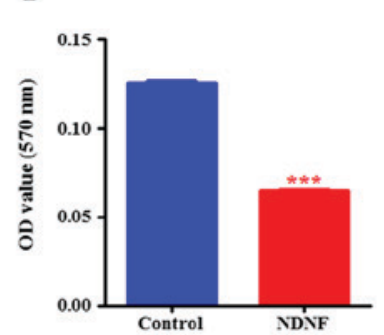

C

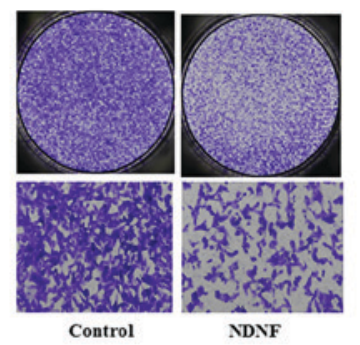

G

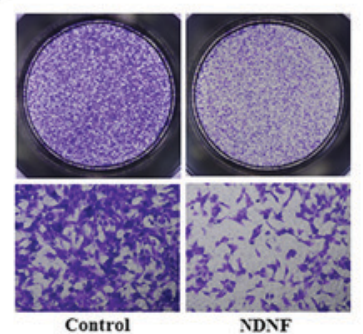

786-O D

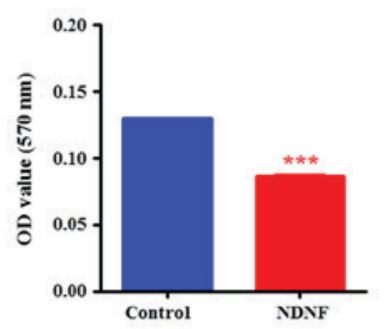

H

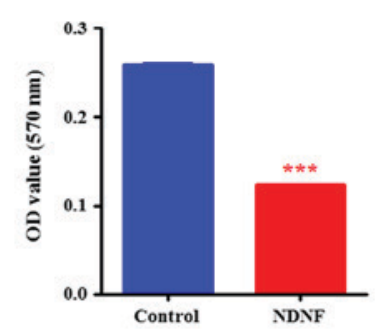

Figure 3. NDNF inhibits the migration and invasion of ACHN (clone 2) and 786-O (clone 3) RCC cells. (A) Representative whole well (x20) and local magnification (x100) image of effects of NDNF on the migration of ACHN cells, as determined by Transwell migration assays. (B) The quantitative analysis of the migrated ACHN cells. (C) Representative whole well (x20) and local magnification (x100) image of effects of NDNF on the migration of 786-O cells, as determined by Transwell migration assays. (D) The quantitative analysis of the migrated 786-O cells. (E) Representative whole well (x20) and local magnification (x100) image of effects of NDNF on the invasion ability of ACHN cells, as determined by Matrigel-coated Transwell invasion assays. (F) The quantitative analysis of the invaded ACHN cells. (G) Representative whole well (x20) and local magnification (x100) image of effects of NDNF on the invasion ability of 786-O cells, as determined by Matrigel-coated Transwell invasion assays. (H) The quantitative analysis of the invaded 786-O cells. All experiments were performed in triplicate. Data are presented as the means $\pm \mathrm{SD}\left(\mathrm{n}=3,{ }^{* * *} \mathrm{P}<0.001\right)$. NDNF group; Lenti-NDNF-infected cells; Control group, Lenti-Control-infected cells. NDNF, neuron-derived neurotrophic factor; OD, optical density; RCC, Renal cell carcinoma; SD, standard deviation.

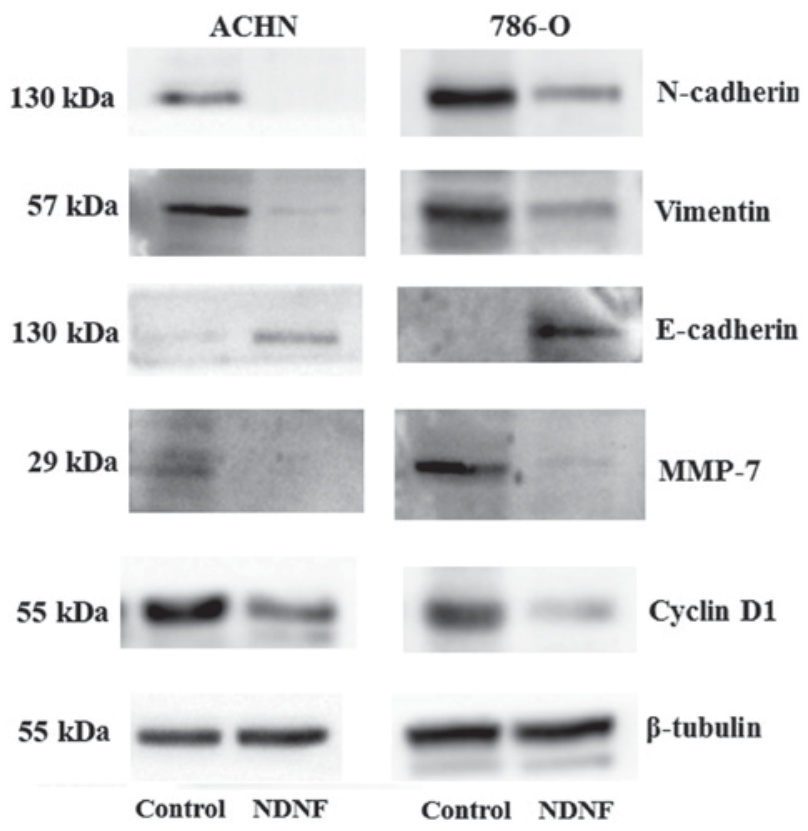

Figure 4. NDNF inhibits EMT in Renal cell carcinoma cells. The protein expression levels of EMT markers and MMP-7 were assessed in ACHN (clone 2) and 786-O (clone 3) cells infected with NDNF, and in control cells, by western blotting. $\beta$-tubulin served as a loading control. NDNF group; Lenti-NDNF-infected cells; Control group, Lenti-Control-infected cells. EMT, epithelial-mesenchymal transition; MMP-7, matrix metalloproteinase 7; NDNF, neuron-derived neurotrophic factor.

with control cells (Fig. 3E-H). Similar results were obtained from ACHN (clone 1) and 786-O (clone 2) cells (data not shown).
NDNF alters the expression of EMT markers in RCC cells. EMT serves a critical role in tumor progression by transforming epithelial cells into a mesenchymal state (11). To determine whether NDNF inhibits RCC invasion via regulating EMT, the expression levels of the epithelial marker E-cadherin, and the mesenchymal markers N-cadherin and vimentin, were examined. Western blotting demonstrated that ectopic expression of NDNF induced a marked increase expression in E-cadherin in ACHN and 786-O cells; however, N-cadherin and Vimentin expression was decreased (Fig. 4).

Since membrane-type matrix metalloproteinases (MT-MMPs) are pivotal regulators of cell invasion, growth and survival, the effects of NDNF on MT-MMP expression were examined. The results revealed that ectopic expression of NDNF markedly inhibited MMP-7 expression (Fig. 4).

\section{Discussion}

Although neurons are considered the primary source of NDNF $(8,9)$, a previous study revealed that NDNF is expressed in normal renal tissues and is downregulated in ccRCC (19). In the present study, NDNF expression was analyzed in $69 \mathrm{ccRCC}$ samples and paired noncancerous tissues, and in silico gene expression data screening was conducted using the Oncomine database (16-18). The present study confirmed that NDNF was decreased in ccRCC. The molecular weight of the strongest band for NDNF was determined at $72 \mathrm{kD}$, although additional unspecific bands were visible. Whether these unspecific bands are modifications of the NDNF protein remains unknown. In addition, NDNF antibody available was used only for western blot analysis and is not suitable for immunohistology staining. 
The expression of NDNF in patients with RCC cannot be correlated with clinical data, e.g. tumor status, grading and survival. The expression of NDNF in a panel of RCC cell lines was also assessed. No NDNF protein was detected in the RCC cells, although NDNF mRNA expression was high in Caki- 1 and Caki- 2 cells. This lack of protein detection could be due to a frameshift mutation or deletion of the NDNF gene, translational regulation via microRNAs or post-translational modifications. These results suggested that the reduced NDNF expression may be associated with RCC carcinogenesis.

To investigate the role of NDNF in RCC carcinogenesis, functional assays were performed. The results demonstrated that restoring NDNF expression decreased the cell proliferation rate by $\sim 20 \%$, and inhibited the migration and invasion rates by $>40 \%$. These data demonstrated that restoring the expression of NDNF markedly suppressed RCC cell proliferation, migration and invasion in vitro, although they were in contradiction with the data observed in neurons (8) and hypoxic endothelial cells (9). This observation may reflect the tissue-specificity of NDFN. It has been reported that some oncogene proteins in glioma may act as tumor suppressors in other types of carcinoma (20-22). In addition, it was demonstrated that restoring NDNF expression could inhibit Cyclin D1 expression in ACHN and 786-O RCC cells. Cyclin D1 is able to form active complexes with either cyclin-dependent kinase (CDK) 4 or CDK6, which, in turn, drives $G_{1}$ to $S$ phase progression (23). NDNF may therefore inhibit RCC cell proliferation by causing $\mathrm{G}_{1}$ to $\mathrm{S}$ phase cell cycle arrest.

The EMT is a normal process associated with organ development and disease, including tumor growth and aggressiveness (7,24,25). During EMT, epithelial cells become more motile and invasive, which are characteristics of mesenchymal-like cells $(25,26)$. It is generally accepted that EMT contributes to tumor development and progression. In the present study, it was demonstrated that restoring NDNF expression reduced the expression levels of some hallmarks of mesenchymal cells, including $\mathrm{N}$-cadherin and Vimentin, but increased E-cadherin. In addition, ectopic NDNF expression significantly inhibited the expression of MMP-7, which is known to cleave E-cadherin (27). It has been reported that MMP-7 is a downstream target gene of Wnt/ $\beta$-catenin signaling, and has an important role in early tumor progression and metastasis (28). Reducing NDNF expression may therefore induce RCC invasion and stimulate EMT via the $\mathrm{Wnt} / \beta$-catenin signaling pathway. However, NDNF ectopic expression has a minor effect on the nuclear localization of $\beta$-catenin (data not shown). The effects of NDNF on RCC cell migration and invasion may therefore involve other pathways, including the Ras signaling pathway; these other pathways should be investigated in future studies.

In conclusion, NDNF was significantly downregulated in ccRCC tissues. In addition, restoring NDNF expression inhibited RCC proliferation, migration and invasion. Ectopic NDNF expression also inhibited RCC progression by suppressing the EMT process. NDNF may therefore act as a regulator of EMT in RCC progression and may represent a novel therapeutic target for the treatment of advanced RCC.

\section{Acknowledgements}

Not applicable.

\section{Funding}

This study was supported by grants from the National Natural Science Foundation of China (grant no. 81472584), the National Key Scientific Program of China (grant no. 2014CBA02005), the Guangdong Key Laboratory funds of Systems Biology and Synthetic Biology for Urogenital Tumors (grant no. 2017B030301015) and the Shenzhen Project of Science and Technology (grant no. 20160520174730707).

\section{Availability of data and materials}

The datasets used and/or analyzed during the present study are available from the corresponding author on reasonable request.

\section{Authors' contributions}

ZL conceived and designed the study. LX, YL, HM, YH, LW and SL developed the methodology and performed experiments; LX and BN analyzed the data; ZC, XL and ZL reviewed and interpreted the data; ZL supervised the study and LX and ZL wrote the manuscript. All authors read and approved the final manuscript.

\section{Ethics approval and consent to participate}

This research was approved by the Shenzhen Second People's Hospital Ethics Committee and was conducted according to Institutional Review Committee guidelines. Written informed consent was obtained from patients.

\section{Patient consent for publication}

Not applicable.

\section{Competing interests}

The authors declare that they have no competing interests.

\section{References}

1. Siegel RL, Miller KD and Jemal A: Cancer statistics, 2016. CA Cancer J Clin 66: 7-30, 2016.

2. Cohen HT and McGovern FJ: Renal-cell carcinoma. N Engl J Med 353: 2477-2490, 2005.

3. Das M: Cabozantinib improves clinical outcomes in renal cancer. Lancet Oncol 18: e2, 2017.

4. Liu S, Tian Z, Zhang L, Hou S, Hu S, Wu J, Jing Y, Sun H, Yu F, Zhao L, et al: Combined cell surface carbonic anhydrase 9 and CD147 antigens enable high-efficiency capture of circulating tumor cells in clear cell renal cell carcinoma patients. Oncotarget 7: 59877-59891, 2016.

5. Xu G, Lu X, Huang T and Fan J: ARHGAP24 inhibits cell cycle progression, induces apoptosis and suppresses invasion in renal cell carcinoma. Oncotarget 7: 51829-51839, 2016.

6. El-Mokadem I, Kidd T, Pratt N, Fleming S and Nabi G: Tumour suppressor gene (CDKNA2) status on chromosome $9 p$ in resected renal tissue improves prognosis of localised kidney cancer. Oncotarget 7: 73045-73054, 2016.

7. Tang H, Chen Y, Liu X, Wang S, Lv Y, Wu D, Wang Q, Luo M and Deng H: Downregulation of HSP60 disrupts mitochondrial proteostasis to promote tumorigenesis and progression in clear cell renal cell carcinoma. Oncotarget 7: 38822-38834, 2016.

8. Kuang XL, Zhao XM, Xu HF, Shi YY, Deng JB and Sun GT: Spatio-temporal expression of a novel neuron-derived neurotrophic factor (NDNF) in mouse brains during development. BMC Neurosci 11: 137, 2010. 
9. Ohashi K, Enomoto T, Joki Y, Shibata R, Ogura Y, Kataoka Y, Shimizu Y, Kambara T, Uemura Y, Yuasa D, et al: Neuron-derived neurotrophic factor functions as a novel modulator that enhances endothelial cell function and revascularization processes. J Biol Chem 289: 14132-14144, 2014.

10. Joki Y, Ohashi K, Yuasa D, Shibata R, Kataoka Y, Kambara T, Uemura Y, Matsuo K, Hayakawa S, Hiramatsu-Ito M, et al: Neuron-derived neurotrophic factor ameliorates adverse cardiac remodeling after experimental myocardial infarction. Circ Heart Fail 8: 342-351, 2015.

11. Dongre A and Weinberg RA: New insights into the mechanisms of epithelial-mesenchymal transition and implications for cancer. Nat Rev Mol Cell Biol: 20 Nov, 2018 (Epub ahead of print).

12. Xie J, Zhu C, Wu J, Li C, Luo L, Xia L, Li X, Gui Y, Cai Z and Li Z: Down-regulation of C12orf59 is associated with a poor prognosis and VHL mutations in renal cell carcinoma. Oncotarget 7: 6824-6834, 2016.

13. Luo L, Wu J, Xie J, Xia L, Qian X, Cai Z and Li Z: Downregulated ECRG4 is associated with poor prognosis in renal cell cancer and is regulated by promoter DNA methylation. Tumour Biol 37 $1121-1129,2016$

14. Livak KJ and Schmittgen TD: Analysis of relative gene expression data using real-time quantitative PCR and the 2(-Delta Delta C(T)) method. Methods 25: 402-408, 2001

15. Rhodes DR, Kalyana-Sundaram S, Mahavisno V, Varambally R, Yu J, Briggs BB, Barrette TR, Anstet MJ, Kincead-Beal C, Kulkarni P, et al: Oncomine 3.0: Genes, pathways, and networks in a collection of 18,000 cancer gene expression profiles. Neoplasia 9: 166-180, 2007.

16. Gumz ML, Zou H, Kreinest PA, Childs AC, Belmonte LS, LeGrand SN, Wu KJ, Luxon BA, Sinha M, Parker AS, et al: Secreted frizzled-related protein 1 loss contributes to tumor phenotype of clear cell renal cell carcinoma. Clin Cancer Res 13 4740-4749, 2007.

17. Lenburg ME, Liou LS, Gerry NP, Frampton GM, Cohen HT and Christman MF: Previously unidentified changes in renal cell carcinoma gene expression identified by parametric analysis of microarray data. BMC Cancer 3: 31, 2003.
18. Yusenko MV, Kuiper RP, Boethe T, Ljungberg B, van Kessel AG and Kovacs G: High-resolution DNA copy number and gene expression analyses distinguish chromophobe renal cell carcinomas and renal oncocytomas. BMC Cancer 9: 152, 2009.

19. Zhou L, Chen J, Li Z, Li X, Hu X, Huang Y, Zhao X, Liang C, Wang Y, Sun L, et al: Integrated profiling of microRNAs and mRNAs: microRNAs located on Xq27.3 associate with clear cell renal cell carcinoma. PLoS One 5: e15224, 2010.

20. Pickard A and McCance DJ: IGF-binding protein 2-oncogene or tumor suppressor? Front Endocrinol (Lausanne) 6: 25, 2015.

21. Echizen K, Nakada M, Hayashi T, Sabit H, Furuta T, Nakai M, Koyama-Nasu R, Nishimura Y, Taniue K, Morishita Y, et al: PCDH10 is required for the tumorigenicity of glioblastoma cells. Biochem Biophys Res Commun 444: 13-18, 2014.

22. Li Z, Chim JC, Yang M, Ye J, Wong BC and Qiao L: Role of PCDH10 and its hypermethylation in human gastric cancer. Biochim Biophys Acta 1823: 298-305, 2012.

23. Musgrove EA, Caldon CE, Barraclough J, Stone A and Sutherland RL: Cyclin D as a therapeutic target in cancer. Nat Rev Cancer 11: 558-572, 2011.

24. Galichon P, Finianos S and Hertig A: EMT-MET in renal disease: Should we curb our enthusiasm? Cancer Lett 341: 24-29, 2013.

25. Piva F, Giulietti M, Santoni M, Occhipinti G, Scarpelli M, Lopez-Beltran A, Cheng L, Principato G and Montironi R: Epithelial to mesenchymal transition in renal cell carcinoma: Implications for cancer therapy. Mol Diagn Ther 20: 111-117, 2016.

26. Han WQ,Zhu Q,Hu J,Li PL,Zhang F and Li N: Hypoxia-inducible factor prolyl-hydroxylase-2 mediates transforming growth factor beta 1-induced epithelial-mesenchymal transition in renal tubular cells. Biochim Biophys Acta 1833: 1454-1462, 2013.

27. McGuire JK, Li Q and Parks WC: Matrilysin (matrix metalloproteinase-7) mediates E-cadherin ectodomain shedding in injured lung epithelium. Am J Pathol 162: 1831-1843, 2003.

28. Tan RJ, Zhou D, Zhou L and Liu Y: Wnt// $/$-catenin signaling and kidney fibrosis. Kidney Int Suppl (2011) 4: 84-90, 2014. 